\title{
Corrección de entropión en párpado inferior con toxina botulínica
}

\section{Lower lid entropion correction using botulinum toxin}

José E. Telich-Tarriba ${ }^{1}$, Alejandra Martínez-Schulte², Ayhdé Grajeda-Gómez ${ }^{2}$, Alejandro Barquet-Fuentes ${ }^{3} y$ José Telich-Vidal**

${ }^{1}$ Departamento de Cirugía Plástica y Reconstructiva, Hospital General Dr. Manuel Gea González; 2Médico Cirujano, Escuela de Medicina, Universidad Panamericana; ${ }^{3}$ Departamento de Oftalmología, Hospital Ángeles Pedregal; ${ }^{4}$ Profesor Titular de Cirugía Estética, Facultad Mexicana de Medicina, Universidad La Salle/Hospital Ángeles Pedregal. Ciudad de México, México

\section{Resumen}

Introducción: El entropión se define como la inversión del borde palpebral, el cual puede tener múltiples etiologías, sin embargo la forma más habitual de presentación es la asociada al proceso de envejecimiento. Esta condición adquiere relevancia debido a las complicaciones oftalmológicas y estéticas que puede desencadenar. Las intervenciones quirúrgicas son las modalidades estándar de tratamiento, sin embargo, varios factores pueden obstaculizar su aplicación. Por esta razón se han desarrollado procedimientos temporales y de mínima invasión para aliviar los síntomas y reducir las complicaciones, entre ellos el uso de la toxina botulínica. Caso clínico: Presentamos el caso de una mujer de 82 años con diagnóstico de entropión involutivo quien rechazó el tratamiento quirúrgico, por lo que se llevó a cabo la aplicación de 12 unidades de toxina botulínica $A$ en el párpado inferior,obteniendo resolución del cuadro durante 16 semanas. Discusión y conclusiones: La toxina botulínica es una alternativa viable, accesible y eficaz para el manejo temporal del entropión, especialmente en pacientes que no deseen o puedan ser candidatos a procedimientos quirúrgicos.

Palabras clave: Toxina botulínica. Entropión. Párpado inferior.

\begin{abstract}
Introduction: Entropion is defined as an inversion of the lower lid border, it may have several etiologies, however the most common form of presentation is associated with the aging process. This clinical condition is mostly relevan due to the ophthalmological and aesthetic complications it may unleash. Surgical procedures are the standard form of management, nevertheless, several factors may hinder their use. For this reason minimally invasive or temporary procedures have been developed to treat this deformity, among them the use of botulinum toxin has been reported. Case report: We present the case of an 82-year-old female diagnosed with involutional entropion who rejected any surgical procedures. After failing conservative measures 12 units of botulinum toxin were applied in the lower lid, obtaining resolution of the entropion for a period of 16 weeks. Discussion and conclusions: Botulinum toxin is a viable, accesible and effective alternative for the temporary management of involutional entropion, especially in patient do not wish to or cannot be subjected to surgical procedures.
\end{abstract}

Key words: Botulinum toxin. Entropion. Lower lid. 


\section{Introducción}

El entropión se define como una posición anómala del párpado, en la cual existe una inversión del borde palpebral, lo que conlleva al roce continuo entre las pestañas y la córnea, o la conjuntiva bulbar. El entropión puede ser de origen congénito, cicatricial, espástico 0 asociado al proceso de envejecimiento (senil o involutivo), siendo esta última forma de presentación la más común en el párpado inferior ${ }^{1}$.

La fisiopatología del entropión involutivo es compleja, se ha postulado que es causada por la combinación de cambios tisulares asociados al proceso degenerativo de la edad, entre los que destacan la laxitud horizontal y vertical del párpado inferior, el predominio de la porción preseptal del músculo orbicular sobre la porción pretarsal, enoftalmos y cambios degenerativos de la lámina tarsal. Clínicamente los pacientes presentan un fórnix inferior profundo, borde palpebral inferior más alto de lo normal y disminución en el rango de movimiento del párpado inferior al dirigir la mirada hacia abajo ${ }^{1-3}$.

La importancia del entropión radica en su sintomatología incapacitante, la cual puede abarcar desde molestias oculares leves, ulceración corneal, queratitis bacteriana, vascularización corneal, hasta pérdida de la visión, así como las alteraciones estéticas que desencadena ${ }^{4}$.

El tratamiento definitivo es quirúrgico; sin embargo en algunas ocasiones debido al riesgo quirúrgico o al rechazo de procedimientos invasivos por parte del paciente se han buscado alternativas de mínima invasión para su tratamiento, entre las cuales destaca el uso de la toxina botulínica tipo $\mathrm{A}^{3,4}$.

Presentamos el caso de una paciente con entropión del párpado inferior con resultados exitosos posterior a la aplicación de toxina botulínica.

\section{Caso clínico}

Femenino de 82 años quien presentó epifora, fotofobia y sensación de cuerpo extraño en el ojo derecho de 3 semanas de evolución. A la exploración física se encontró hiperemia conjuntival, queratitis e inversión del párpado inferior, sin ulceraciones corneales (Fig. 1).

La paciente fue diagnosticada con entropión involutivo, sin embargo rechazó el tratamiento quirúrgico por lo que fue manejada sin éxito con lubricantes y parche ocular nocturno. Ante la falta de mejoría, el servicio de cirugía plástica de nuestra institución fue consultado y se propuso la aplicación de toxina botulínica tipo $A$ como medida de corrección temporal del entropión.

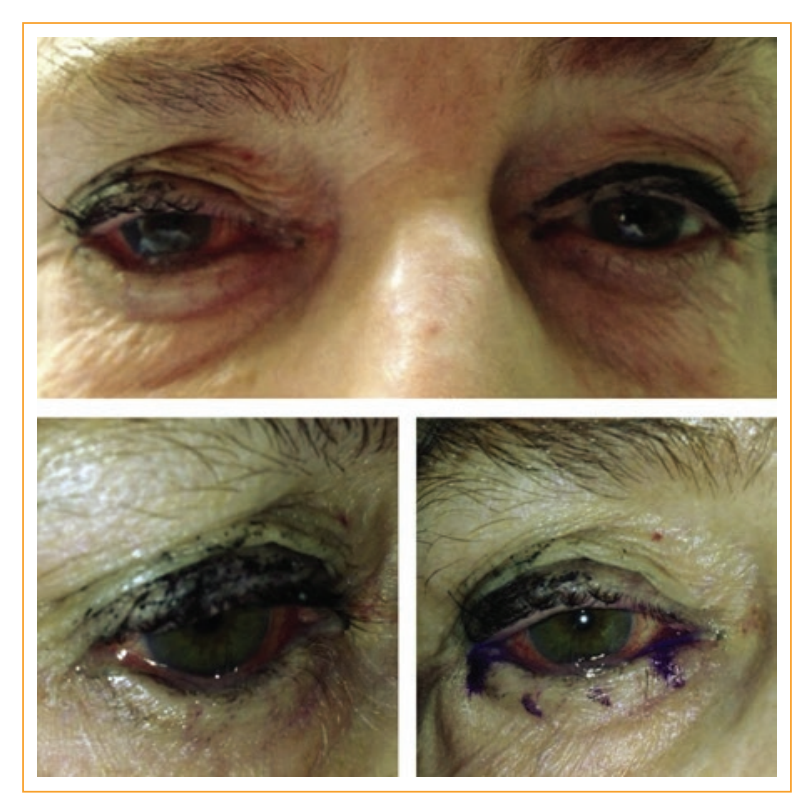

Figura 1. Paciente con entropión degenerativo en párpado inferior derecho. Nótese la inversión de pestañas e irritación conjuntival.

Se aplicaron un total de 12 unidades de toxina $\left(B^{\circ}\right.$ toto $^{\circledR}$, Allergan Inc., EE. UU.) vía subcutánea sobre la porción pretarsal del músculo orbicular del ojo derecho en 4 puntos $3 \mathrm{~mm}$ por debajo del reborde palpebral.

Una semana después la paciente presentó resolución del entropión, con eversión del borde palpebral y sin contacto entre las pestañas y el globo ocular (Fig. 2), el efecto tuvo una duración de 16 semanas, tras lo cual el entropión reapareció gradualmente, llevando al equipo a aplicar nueva dosis de toxina botulínica $A$. No se presentaron complicaciones ni efectos adversos.

\section{Discusión}

A pesar de que la cirugía continúa siendo el estándar de oro para la corrección del entropión ${ }^{2}$ se ha visto que pueden desarrollarse complicaciones corneales durante el tiempo de espera para el procedimiento quirúrgico, por lo que se han desarrollado medidas conservadoras para evitarlas, como el uso de lubricantes oftálmicos, la oclusión temporal con parches y el uso de la toxina botulínica ${ }^{1,4,5}$, la cual ha surgido como una alternativa viable, especialmente para aquellos pacientes que no son candidatos o rechazan los procedimientos invasivos ${ }^{3}$.

La toxina botulínica $A$ es una exotoxina producida por Clostridium botulinum, un bacilo grampositivo anaerobio. Su mecanismo de acción consiste en la creación de una parálisis flácida, al inhibir la liberación de 


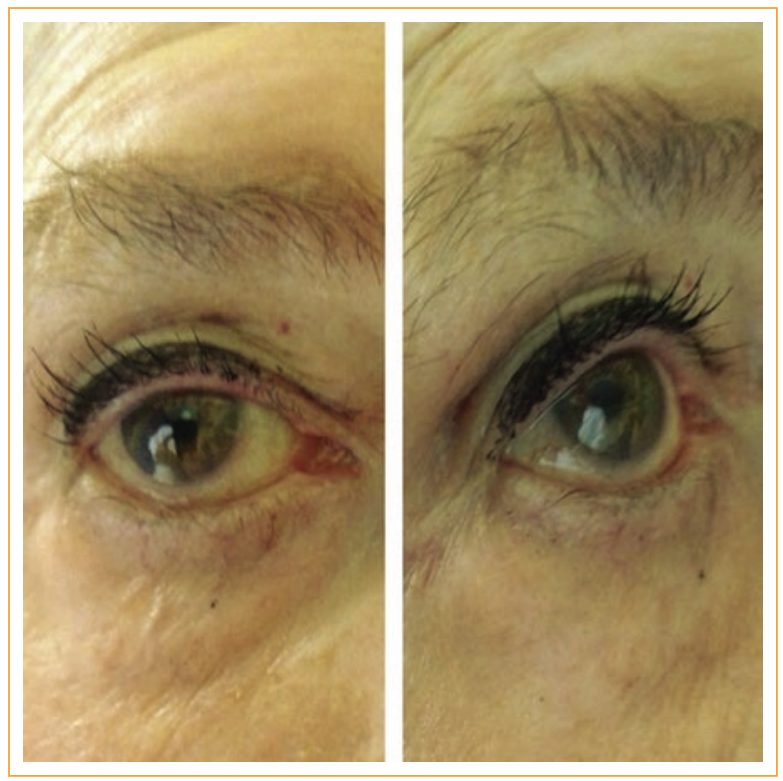

Figura 2. Evolución de la paciente 3 semanas postaplicación de toxina botulínica. Se evidencia eversión de borde palpebral y pestañas, acompañado por resolución de la irritación conjuntival.

acetilcolina en la placa neuromuscular ${ }^{3,6}$. La toxina fue utilizada por primera vez por Scott et al. en 1973 en pacientes con estrabismo; posteriormente fue aprobado por la FDA para el tratamiento de retracción del parpado superior, regeneración aberrante del nervio facial, hipersecreción de la glándula lacrimal, síndrome de ojo seco, arrugas faciales dinámicas, entre otros ${ }^{7}$.

El uso de la toxina botulínica en el manejo del entropión involutivo fue descrito por primera vez en 1988 por Clarke et al.; a partir de entonces se han reportado series pequeñas con resultados satisfactorios. Teóricamente la resolución del entropión se logra al ejercer acción sobre la porción preseptal del músculo orbicular, sin afectar la laxitud tarsal y el retractor palpebral ${ }^{4}$. La recuperación de la acción del músculo ocurre por brote axonal y formación de nuevas uniones neuromusculares ${ }^{2}$.

En el caso de nuestra paciente se propone un tratamiento no quirúrgico para la mejoría clínica de signos y síntomas mediante la aplicación de toxina botulínica A directamente sobre el músculo orbicular hipertrofiado. Se demostró que el efecto de la toxina mejora el pliegue cutáneo, el roce de las pestañas a la cornea desaparece de forma gradual, desde la primera semana hasta el cuarto mes como se esperaría encontrar, ya que se ha reportado que la parálisis flácida que produce la toxina botulínica $A$ inicia entre las 24 a 48 horas con un pico máximo entre los 7 a
10 días, y con duración del efecto entre 12 a 15 semanas $^{3,4,8-10}$.

Una de las ventajas de este procedimiento es la simplicidad, ya que es un procedimiento corto, relativamente sencillo, de mínima invasión y donde se han descrito escasas complicaciones (lagoftalmos, ectropión o entropión, epifora funcional por falla del saco lacrimal, diplopía y hematoma del parpado), además no ocasiona cambios a largo plazo en la morfología del músculo orbicular ${ }^{4,7-10}$. Tal es el caso de nuestra paciente que no presentó ninguna complicación o reacción adversa.

\section{Conclusiones}

La toxina botulínica es una alternativa viable, accesible y eficaz para el manejo temporal del entropión, especialmente en pacientes que no deseen o puedan ser candidatos a procedimientos quirúrgicos, de esta forma se disminuye la probabilidad de complicaciones corneales, así como el alivio de la sintomatología.

\section{Responsabilidades éticas}

Protección de personas y animales. Los autores declaran que los procedimientos seguidos se conformaron a las normas éticas del comité de experimentación humana responsable y de acuerdo con la Asociación Médica Mundial y la Declaración de Helsinki.

Confidencialidad de los datos. Los autores declaran que han seguido los protocolos de su centro de trabajo sobre la publicación de datos de pacientes.

Derecho a la privacidad y consentimiento informado. Los autores han obtenido el consentimiento informado de los pacientes y/o sujetos referidos en el artículo. Este documento obra en poder del autor de correspondencia.

\section{Financiamiento}

Los autores no recibieron patrocinio para llevar a cabo este artículo.

\section{Conflicto de intereses}

Los autores declaran no tener ningún conflicto de intereses.

\section{Bibliografía}

\footnotetext{
1. Pereira MG, Rodrigues MA, Rodrigues SA. Eyelid entropion. Semin Ophtalmol. 2010;25:52-8.

2. Deka A, Saikia SP. Botulinum toxin for lower lid entropion correction.
} Orbit. 2011;30:40-2. 
Rev Mex Oftalmol. 2018;92

3. Clarke JR, Spalton DJ. Treatment of senile entropion with botulinum toxin. Br J Ophtalmol. 1988;72:361-2.

4. Steel DH, Hoh HB, Harrard RA, et al. Botulinum toxin for the temporary treatment of involutional lower lid entropion: A clinical and morphological study. Eye (Lond). 1997;11:472-5.

5. Irvine S, Francis IC, Bishop AE, et al. The entropion patch: A method of temporarily correcting involutional entropion with adhesive tape. Ophthalmic Surg. 1994;25:604-6.

6. Christiansen G, Mohney B, Baratz H, et al. Botulinum toxin for the treatment of congenital entropion. Am J Ophtalmol. 2004;138:153-5.
7. Naik MN, Soparkar CN, Murthy R, Honavar SG. Botulinum toxin in ophthalmic plastic surgery. Indian J Ophthalmol. 2005;53:279-8.

8. Kaynak-Hekimhan P. Noncosmectic periocular therapeutic applications of botulinum toxin. Middle East Afr J Ophthalmol. 2010;17:113-20.

9. Magoon EH. Botulinum toxin for treatment of blepharospasm, corneal exposure, and entropion. J Ocul Ther Surg. 1985;4:133-5

10. Cillino S, Raimondi G, Guépratte N, et al. Long- term efficacy of botulinum toxin A for treatment of blepharospasm, hemifacial spasm and spastic entropion: A multicentre study using two drug dose escalation indexes. Eye (Lond). 2010;24:600-7. 